\title{
Multiple sound reflections between plane panels covered with reflection coefficients
}

\author{
Yoshimasa Sakurai and Koji Ishida \\ Faculty of Engineering, Kansai University, \\ 3-3-35, Yamate-cho, Suita, Osaka, 564 Japan
}

(Received 7 June 1982)

\begin{abstract}
The sound reflection coefficient of a given material, which is measured from a sufficiently large plane of it for spherical wave incidence, has been used previously to predict the first reflection for plane, convex or concave, finite size panels covered with that material. The first reflection of such a panel can be treated as two separate waves. One is the wave from the specular reflection point and the other is a boundary wave comprised of components from the lined point sources at each edge. Here, multiple reflections between two plane panels covered with materials having reflection coefficients that may be different are approximately calculated from such separate waves as in the same treatment of rigid plane panels.
\end{abstract}

PACS number: $43.55 . \mathrm{Br}$

\section{CALCULATION OF THE MULTIPLE REFLECTIONS BETWEEN TWO PLANE PANELS WITH REFLECTION COEFFICIENTS}

A first reflection $\varphi_{1}(Q)$ of a plane panel $F_{1}$ covered with a material having a reflection coefficient to a point $Q$ on a panel $F_{2}$ in Fig. 1 is approximated ${ }^{1)}$ as in the following,

$$
\varphi_{1}(\mathrm{Q})=R_{\omega 10} \iint_{\mathrm{F}_{1}} K_{\omega} \cdot d \mathrm{~F}=R_{\omega 10} \cdot \varphi_{10}(\mathrm{Q}),
$$

where $R_{\omega 10}$ is a reflection coefficient at the specular reflection point on the panel $F_{1}$ and $\varphi_{10}(Q)$ is the reflection of the rigid plane panel ${ }^{2)}$ at the panel $F_{1}$. The second reflection $\varphi_{\omega}(\mathbf{P})$ at $\mathbf{P}$ from the panel $F_{2}$ is obtained by substituting Eq. (1) into Helmholtz-Kirchhoff's formula as in the following,

$$
\begin{aligned}
\varphi_{\omega}(\mathrm{P})= & \frac{1}{4 \pi} \iint_{\mathrm{F}_{2}}\left\{R_{\omega 2} \cdot \varphi_{1}(\mathrm{Q}) \frac{\partial}{\partial n}\left(\frac{e^{-i k l}}{l}\right)\right. \\
& \left.-\frac{\partial}{\partial n}\left(R_{\omega 2} \cdot \varphi_{1}(\mathrm{Q})\right) \frac{e^{-i k l}}{l}\right\} d \mathrm{~F}_{2}
\end{aligned}
$$

where $n$ is an inward normal and $R_{\omega_{2}}$ is a reflection coefficient on the panel $F_{2}$. The single layer term in
Eq. (2) is separated as

$$
\begin{aligned}
& \frac{\partial}{\partial n}\left(R_{\omega 2} \cdot \varphi_{1}(\mathrm{Q})\right) \frac{e^{-i k l}}{l} \\
& \quad=\left\{\frac{\partial R_{\omega 2}}{\partial n} \varphi_{1}(\mathrm{Q})+R_{\omega 2} \frac{\partial \varphi_{1}(\mathrm{Q})}{\partial n}\right\} \frac{e^{-i k l}}{l} .
\end{aligned}
$$

A first reflection $\varphi_{1}(\mathrm{Q})$ in Eq. (1) is composed of two kinds of sound sources. One of them is an image point source with a reflection coefficient and the others are boundary waves. The boundary wave at an edge element $\Delta g$ has a directivity which could be replaced by point sources. When $D_{j}$ is the magnitude of one of them and $\left(m_{j}^{\prime}+l_{j}{ }^{\prime}\right)$ is a distance from the source to a point on the panel $F_{2}$, one of the first reflections $\varphi_{1}(\mathrm{Q})$ in the first term of the right hand side of Eq. (3) is expressed by $R_{\omega 10}$. $D_{j} \cdot \Delta g \cdot \exp \left[-i k\left(m_{j}^{\prime}+l_{j}^{\prime}\right)\right] /\left(m_{j}^{\prime}+l_{j}^{\prime}\right)$. When $R_{\omega 2}$ is a reflection coefficient on the panel $F_{2}$ to this incident wave, the reflection from the second panel $F_{2}$ by the source, which is one of the integrations for the first term in Eq. (3), is given by,

$$
\begin{aligned}
& R_{\omega 10} \cdot D_{j} \cdot \Delta g \iint_{\mathrm{F}_{2}}\left\{\partial R_{\omega 2} / \partial n\right. \\
& \left.\quad \cdot \exp \left[-i k\left(m_{j}^{\prime}+l_{j}^{\prime}+l\right)\right] /\left(m_{j}^{\prime}+l_{j}\right) l\right\} d \mathrm{~F}_{2} .
\end{aligned}
$$




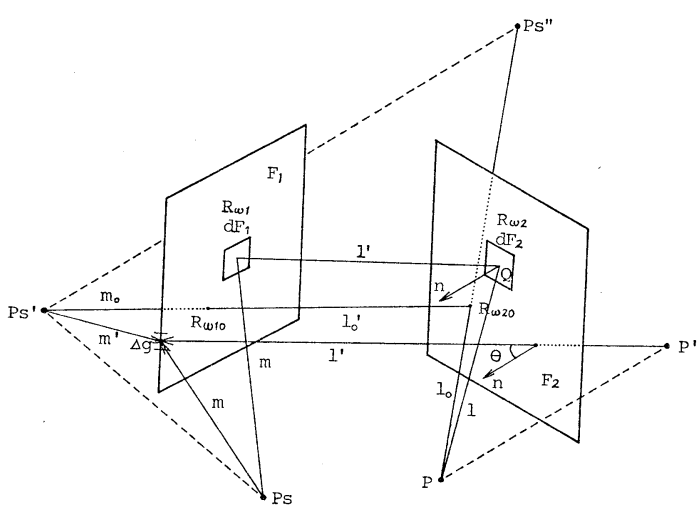

Fig. 1 Multiple reflections between two plane panels covered with materials having reflection coefficients; a point source is at $P_{\mathrm{S}}$.

If $\partial R_{\omega 2} / \partial n$ changes continuously on the panel $F_{2}$ and $\partial R_{\omega 20} / \partial n$ shows its value at the specular reflection, the integration is approximately estimated from the same treatment ${ }^{1)}$ that is applied for the approximation of Eq. (1), as in the following,

$$
\begin{aligned}
& R_{\omega 10} \cdot D_{j} \cdot \Delta g \cdot \partial R_{\omega 20} / \partial n \\
& \quad \cdot \iint_{\mathrm{F}_{2}} \exp \left[-i k\left(m_{j}^{\prime}+l_{j}^{\prime}+l\right)\right] /\left(m_{j}^{\prime}+l_{j}^{\prime}\right) l \cdot d \mathrm{~F}_{2} .
\end{aligned}
$$

It is experimentally shown ${ }^{1 / 2}$ for uniform surfaces such as punch-carpet and urethane foam layers that the partial derivative of a reflection coefficient to the direction of the specular reflection, $\partial R_{\omega 20} / \partial l_{0}{ }^{\prime}$, is nearly equal to zero. $\partial R_{\omega 20} / \partial n$ is almost zero also, because $\partial R_{\omega 20} / \partial l_{0}{ }^{\prime}$ is equal to $\partial R_{\omega 20} / \partial n \cdot \partial n / \partial l_{0}{ }^{\prime}$. Therefore the above term vanishes. Finally, the integration for the first term in the right hand side of Eq. (3) is estimated as nearly zero after the same calculations for the image source and the other point sources substituted for the boundary waves.

Then Eq. (2) becomes as in the following,

$$
\begin{aligned}
\varphi_{\omega}(\mathrm{P})= & \frac{1}{4 \pi} \iint_{\mathrm{F}_{2}} R_{\omega 2}\left\{\varphi_{1}(\mathrm{Q}) \frac{\partial}{\partial n}\left(\frac{e^{-i k l}}{l}\right)\right. \\
& \left.-\frac{\partial \varphi_{1}(\mathrm{Q})}{\partial n} \frac{e^{-i k l}}{l}\right\} d \mathrm{~F}_{2} .
\end{aligned}
$$

This equation has separate incident waves on the panel $F_{2}$. One is the component around the specular reflection point and the other is that of boundary waves. Incident angles on the panel $F_{2}$ from those of the panel $F_{1}$ are different from each other and their multiple reflections are separately estimated.

A second reflection $\varphi_{\omega 1}(\mathrm{P})$ for the component around the specular reflection point on $F_{1}$ is given by,

$$
\begin{aligned}
\varphi_{\omega 1}(\mathrm{P})= & \frac{1}{4 \pi} \iint_{\mathrm{F}_{2}} R_{\omega 10} \cdot R_{\omega 2}\left\{\varphi_{10}(\mathrm{Q}) \frac{\partial}{\partial n}\left(\frac{e^{-i k l}}{l}\right)\right. \\
& \left.-\frac{\partial \varphi_{10}(\mathrm{Q})}{\partial n} \frac{e^{-i k l}}{l}\right\} d \mathrm{~F}_{2}
\end{aligned}
$$

where $\varphi_{10}(\mathrm{Q})$ is a geometrical wave from $F_{1}$. When a reflection coefficient $R_{\omega 2}$ is approximately replaced by $R_{\omega 20}$, which is at the specular reflection point on $F_{2}$, the same consideration as Eq. (1) is applied and those two coefficients are treated as constants, then Eq. (5) is,

$$
\begin{aligned}
\varphi_{\omega 1}(\mathrm{P})= & \frac{R_{\omega 10} \cdot R_{\omega 20}}{4 \pi} \iint_{\mathrm{F}_{2}}\left\{\varphi_{10}(\mathrm{Q}) \frac{\partial}{\partial n}\left(\frac{e^{-i k l}}{l}\right)\right. \\
& \left.-\frac{\partial \varphi_{10}(\mathrm{Q})}{\partial n} \frac{e^{-i k l}}{l}\right\} d \mathrm{~F}_{2} .
\end{aligned}
$$

The integration is the second reflection of a geometrical wave of $F_{1}$ reflected on the rigid plane panel at $F_{2}$, which is estimated in the same way as in the previous paper. ${ }^{3)}$

When a boundary wave at an edge of $F_{1}$ is treated as lined point sources, their specular reflections on $F_{2}$ are the first order of approximation of its second reflections. Though each point source of the boundary waves has a different incident angle onto $F_{2}$, the point source with the shortest path is most predominant, because the component along that path yields the largest boundary wave. A reflection coefficient for a boundary wave is chosen at the incident angle $\theta$ on $\mathrm{F}_{2}$, when such a shortest path from a point source to an image of a receiving point $\mathrm{P}^{\prime}$ crosses as in Fig. 2.

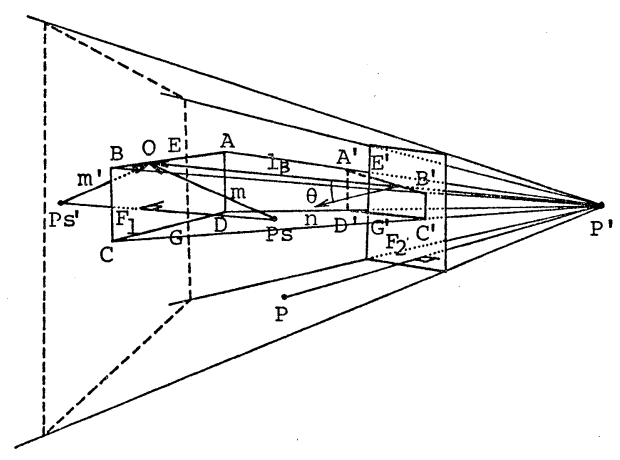

Fig. 2 Specular reflection of a boundary wave whose largest contribution is from the shortest path $\mathrm{P}_{\mathrm{S}}{ }^{\prime} \mathrm{OP}^{\prime}$ on the edge $\mathrm{BE}$ with the incident angle $\theta$ on the panel $F_{2}$. 


\section{Y. SAKURAI and K. ISHIDA: MULTIPLE REFLECTIONS OF PANELS WITH COEFFICIENTS}

When $q$ is the number of edges which make specular reflections on $\mathrm{F}_{2}$ and $R_{\omega 2 j}$ is the reflection coefficient for a boundary wave of an edge $j$ with the shortest path, the specular reflections of boundary waves of the edges, $\varphi_{\omega 2}(\mathrm{P})$, is obtained by,

$$
\begin{aligned}
\varphi_{\omega 2}(\mathrm{P})= & \frac{R_{\omega 10}}{4 \pi} \sum_{j=1}^{q} R_{\omega 2 j} \iint_{\mathrm{F}_{2}}\left\{\varphi_{10 j}(\mathrm{Q}) \frac{\partial}{\partial n}\left(\frac{e^{-i k l}}{l}\right)\right. \\
& \left.-\frac{\partial \varphi_{10 j}(\mathrm{Q})}{\partial n} \frac{e^{-i k l}}{l}\right\} d \mathrm{~F}_{2},
\end{aligned}
$$

where $\varphi_{10 j}(\mathrm{Q})$ is a boundary wave from a rigid plane panel at $F_{1}$ and the integration is estimated by the line integral Eq. (1) with the conditions Eq. (5) of Ref. 3) (see Fig. 2).

The second reflected boundary waves are approximated by Eq. (6), when its integration term calculates the second reflected boundary waves between two rigid plane panels at the same position of these two plane panels. $^{3)}$

\section{COMPARISON OF CALCULATED AND MEASURED RESULTS}

A panel covered with a urethane foam layer of $10 \mathrm{~mm}$ thickness and one with a punch-carpet layer of about $4 \mathrm{~mm}$ thickness are the panels $F_{1}$ and $F_{2}$ in Fig. 3, respectively, where the surfaces are at the same positions. Reflection coefficients from sufficiently large planes covered with those layers in the time domain are shown in Fig. 4(a) and (b). ${ }^{1)}$

Calculated impulse responses of multiple reflections are separately shown in Fig. 5; (i) first reflections by Eq. (1), (ii) second specular reflections by Eq. (6), and (iii) specular reflections of boundary waves by Eq. (7) and second reflected boundary waves by Eq. (6) with $R_{\omega 10}$ and $R_{\omega 20}$ at the specular reflections of the panels. Second reflected boundary waves are negligibly small, compared with the specular reflections of boundary waves. These reflections are superposed in Fig. 6 shown by a dotted line and compared with the measured impulse response shown by a solid line.

The same comparisons for the panels covered with the punch-carpet layers are shown in Figs. 7 and 8 . They agree sufficiently with each other. When one of the panels is covered with a urethane foam layer, they agree better.

Since it is possible to separate multiple reflections from the first reflections in these cases, measured and calculated transfer functions of them are compared in Fig. 9(a) and (b). It is evident that
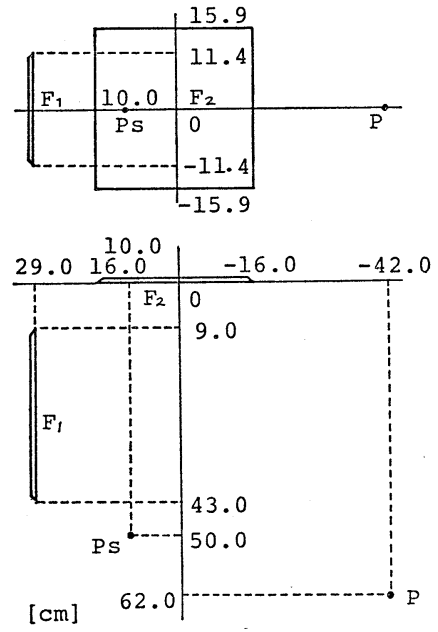

Fig. 3 Two plane panels having reflection coefficients.

the multiple reflections of boundary waves explain the measured results much better. In Fig. 10, measured and calculated transfer functions of the multiple reflections shown in Figs. 6 and 8 are compared (in solid and dotted lines, respectively). There is still a little difference in both domains which are thought to be caused by neglecting the secondary disturbances at the edges, and by the waves diffracted into the backs of the panels.

For smoothly curved panels with reflection coefficients, this method seems to be applicable; since the first reflection can also be estimated ${ }^{1)}$ by Eq. (1), the separate treatment of multiple reflections of the specular reflection and boundary waves for plane panels with reflection coefficients is valid as shown in this paper, and multiple reflections of the specular reflection between rigid curved panels are also discretely predominant. ${ }^{4,5)}$

When a panel is small and is covered with a thick porous layer, Eq. (1) itself can not predict its reflection well, because the secondary disturbances at an edge emerges. When both panels have no specular reflections, reflection coefficients for the boundary waves are replaced by those at the specular reflection points outside the panels. In these cases, this method may not be applicable for the precise calculation of the multiple reflections between those panels. This method, however, might be satisfactory for the calculation of the multiple reflections in the sound field of an auditorium, because their reflec- 


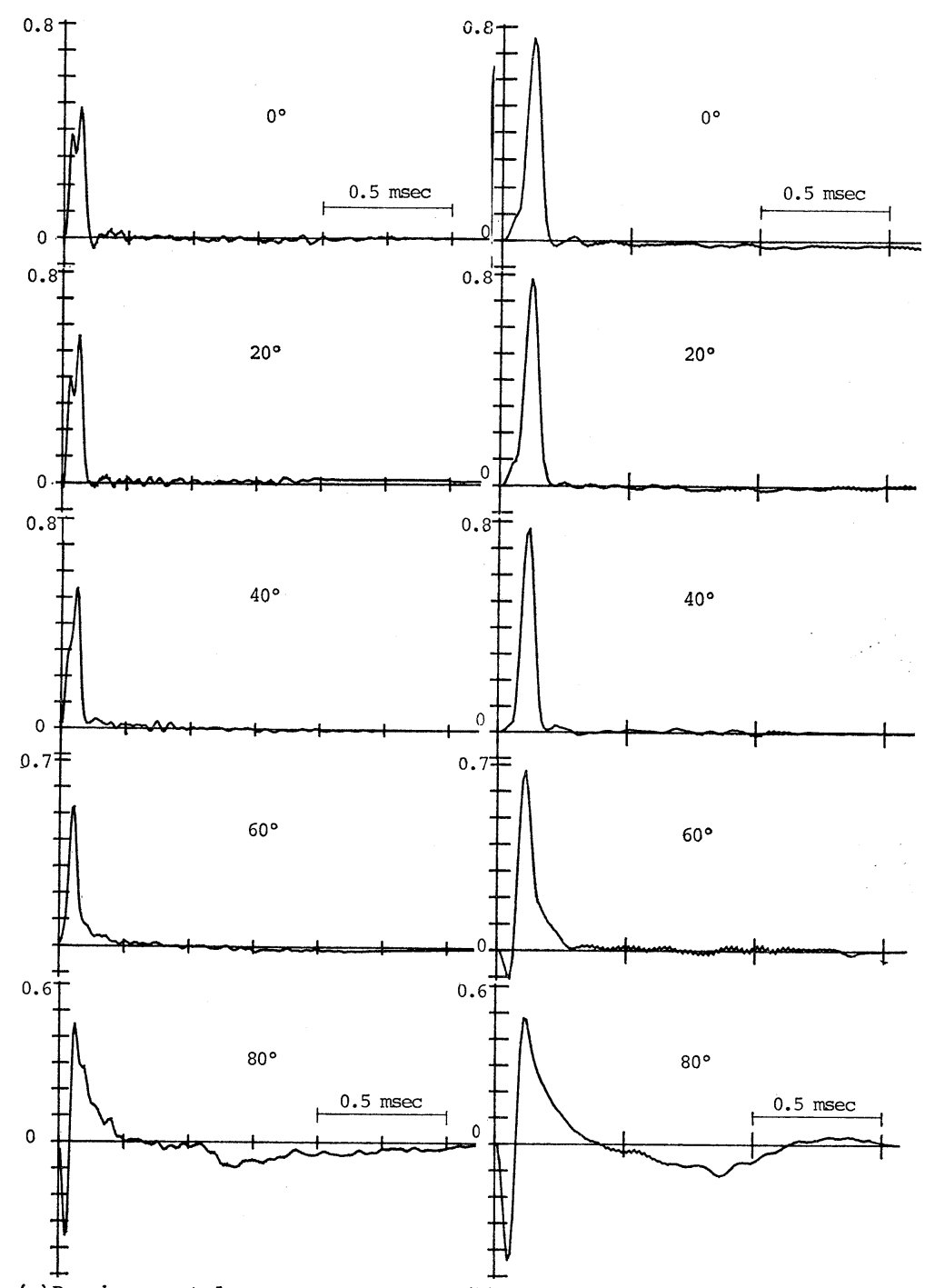

(a) Punch-carpet layer.

(b) Urethane foam layer.

Fig. 4 Reflection coefficients in the time domain of punchcarpet and urethane foam layers. ${ }^{1)}$

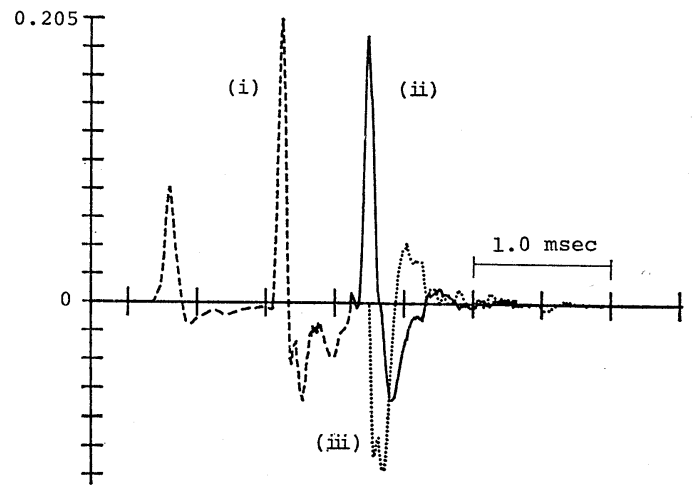

Fig. 5 Calculated impulse response of each reflection for the panels with a urethane foam and a punchcarpet layers; (i) first reflections, (ii) second specular reflections, and (iii) specular reflections of boundary waves and second reflected boundary waves. 


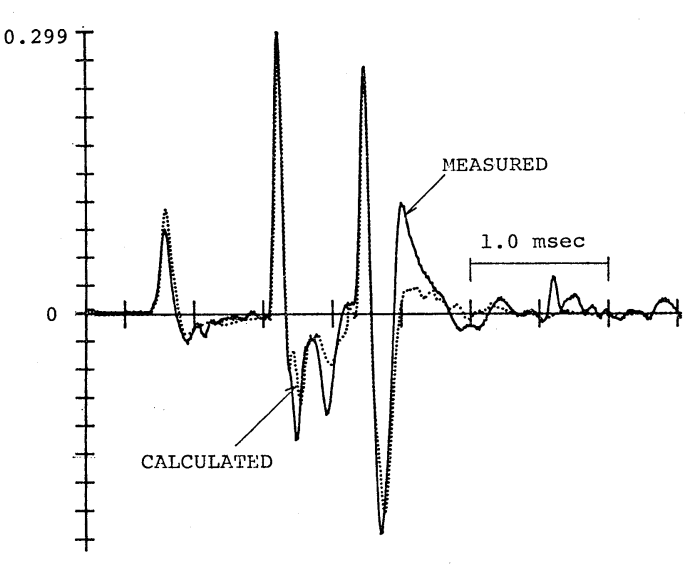

Fig. 6 Measured and calculated impulse responses of multiple reflections between the panels with a urethane foam, and a punch-carpet, layer.

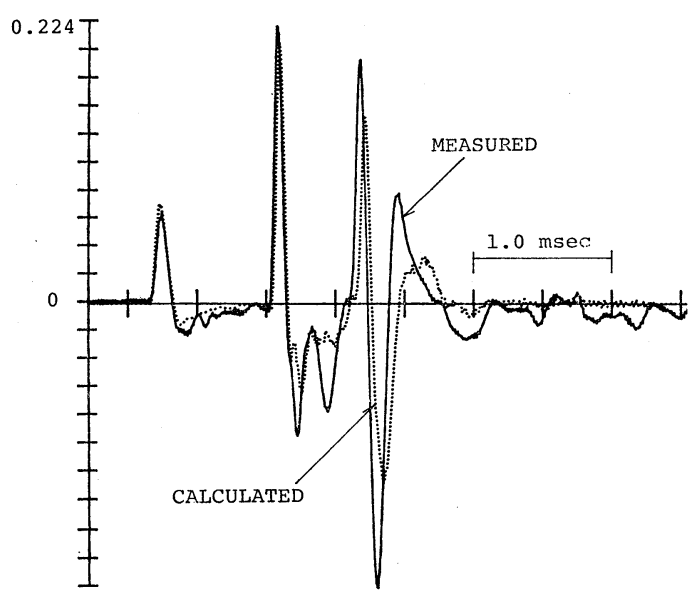

Fig. 8 Measured and calculated impulse responses of multiple reflections between the panels with punch-carpet layers.

tions are usually much less than the others and do not change the transfer function of the early reflections much, giving the approximation of the first order.

\section{CONCLUSIONS}

The calculations of multiple reflections between two plane panels covered with materials having reflection coefficients are estimated separately depending on the reflection paths.

For a specular reflection, the reflection coeffi-

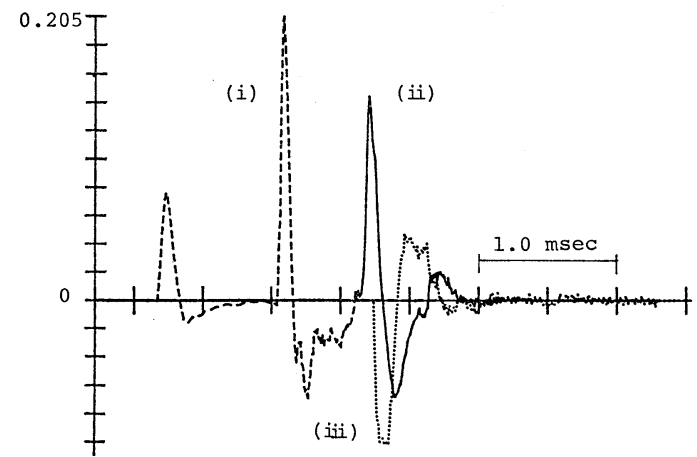

Fig. 7 Calculated impulse response of each reflection for the panels with punch-carpet layers; (i), (ii) and (iii) are the same as in Fig. 5.

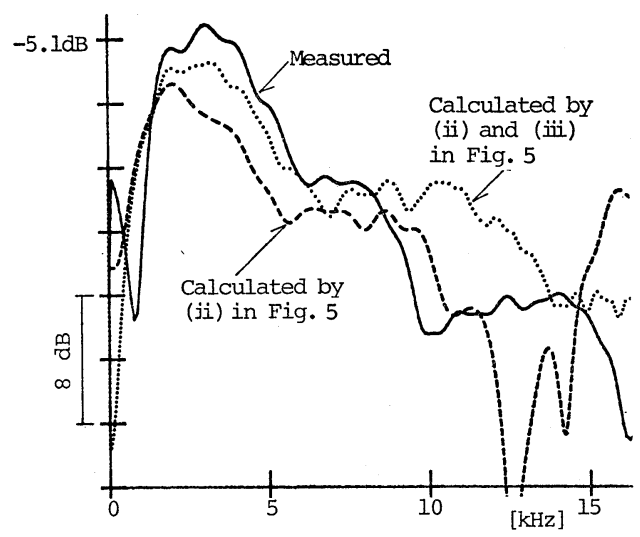

(a) For panels with punch-carpet and urethane foam layers.

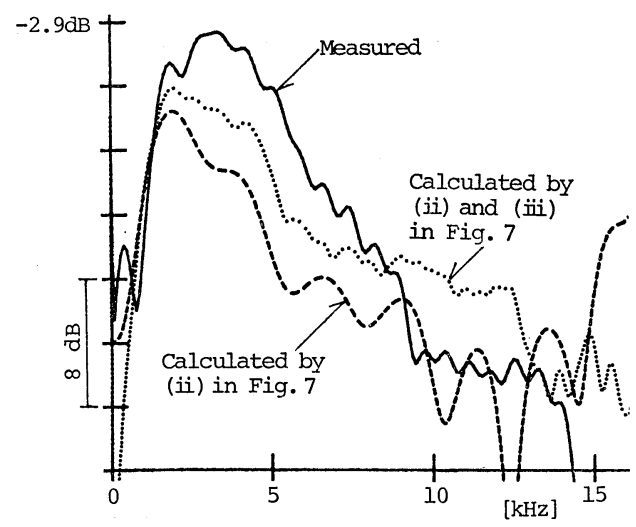

(b) For panels with punch-carpet layers.

Fig. 9 Comparison of measured and calculated transfer functions of the second reflections in; (a) Fig. 6 and (b) Fig. 8. 

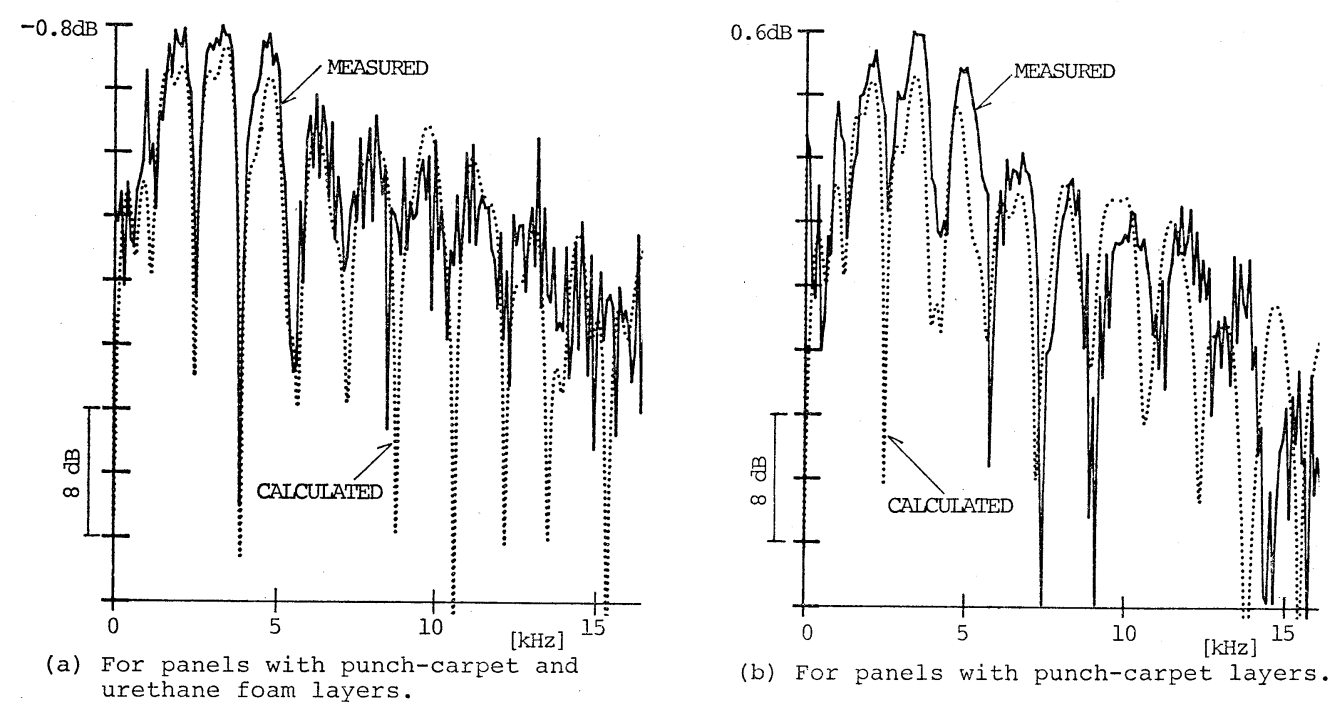

Fig. 10 Comparison of transfer functions of the measured and calculated multiple reflections in;

(a) Fig. 6 and (b) Fig. 8.

cients at the incident angle of the first and second specular reflection points are convoluted with the second reflection of a geometrical wave between two rigid plane panels which are at the same positions of the panels. For a specular reflection of a boundary wave, a reflection coefficient at the incident angle on the second panel which makes the shortest path on an edge from a point source to a receiving point is convoluted with a geometrical reflection of a boundary wave between two rigid plane panels at the same positions of the panels.

The results calculated by the specular reflection and the sum of the reflections of each edge correspond sufficiently with the measured results; and especially the introduction of a specular reflection of a boundary wave explains the measured results better.

These results suggest the possibility of the calculation of the multiple reflection between curved panels covered with materials having reflection coefficients.

\section{ACKNOWLEDGMENTS}

The authors wish to express their appreciation to
Mr. Y. Kawai, teaching and research assistant at Osaka Institute of Technology, for his participation in useful discussions. They also wish to express thanks to Dr. Z. Maekawa, Professor at Kobe University and Dr. K. Matsuura, Professor at Kyoto University, for their continuous encouragement.

\section{REFERENCES}

1) Y. Sakurai and K. Nagata, "Practical estimation of sound reflection of a panel with a reflection coefficient," J. Acoust. Soc. Jpn.(E) 3, 7-19 (1982).

2) Y. Sakurai and K. Nagata, "Sound reflections of a rigid plane panel and of the "live end" composed by those panels," J. Acoust. Soc. Jpn.(E) 2, 5-14 (1981).

3) Y. Sakurai and K. Ishida, "Multiple reflections between rigid plane panels," J. Acoust. Soc. Jpn. (E) 3, 183-190 (1982).

4) Y. Sakurai, "Sound reflection of a curved rigid panel," J. Acoust. Soc. Jpn.(E) 2, 63-70 (1981).

5) Y. Sakurai and K. Ishida, "Multiple reflections between rigid curved panels," J. Acoust. Soc. Jpn. (E) 4, 27-33 (1983). 